\title{
Increased cathepsin $K$ levels in human atherosclerotic plaques are associated with plaque instability
}

\author{
HUIYING ZHAO $^{1}$, XIUJIAO QIN ${ }^{1}$, SHUAI WANG $^{2}$, XIWEI SUN $^{3}$ and BIN DONG ${ }^{1}$ \\ ${ }^{1}$ Department of Gerontology, First Hospital, Jilin University; ${ }^{2}$ Department of Cardiology, Jihua General Hospital; \\ ${ }^{3}$ Department of Vascular Surgery, First Hospital, Jilin University, Changchun, Jilin 130021, P.R. China
}

Received September 28, 2016; Accepted May 16, 2017

DOI: 10.3892/etm.2017.4935

\begin{abstract}
Investigating the determinants and dynamics of atherosclerotic plaque instability is a key area of current cardiovascular research. Extracellular matrix degradation from excessive proteolysis induced by enzymes such as cathepsin $\mathrm{K}(\mathrm{Cat} \mathrm{K})$ is implicated in the pathogenesis of unstable plaques. The current study assessed the expression of $\mathrm{Cat} \mathrm{K}$ in human unstable atherosclerotic plaques. Specimens of popliteal arteries with atherosclerotic plaques were classified as stable $(<40 \%$ lipid core plaque area; $n=6)$ or unstable $(\geq 40 \%$ lipid core plaque area; $n=14$ ) based on histopathological examinations of hematoxylin and eosin stained sections. The expression of Cat $\mathrm{K}$ and cystatin C (Cys C) were assessed by immunohistochemical examination and levels of Cat K mRNA were detected by semi-quantitative reverse transcriptase polymerase chain reaction. Morphological changes including a larger lipid core, endothelial proliferation with foam cells and destruction of internal elastic lamina were observed in unstable atherosclerotic plaques. In unstable plaques, the expression of $\mathrm{Cat} \mathrm{K}$ protein and mRNA was upregulated, whereas Cys $\mathrm{C}$ protein expression was downregulated. The interplay between Cat $\mathrm{K}$ and Cys $\mathrm{C}$ may underlie the progression of plaques from stable to unstable and the current study indicated that Cat $\mathrm{K}$ and Cys $\mathrm{C}$ are potential targets for preventing and treating vulnerable atherosclerotic plaque ruptures.
\end{abstract}

\section{Introduction}

Atherosclerosis is the principal pathological change that occurs in cardiovascular disease, which is a leading cause of mortality and morbidity worldwide (1). Rupture of an unstable atherosclerotic plaque, which is characterized by a thinned-out collagen-depleted fibrous cap, a large lipid-rich necrotic core

Correspondence to: Dr Huiying Zhao, Department of Gerontology, First Hospital, Jilin University, 71 Xinmin Street, Changchun, Jilin 130021, P.R. China

E-mail: zhaohuiying163@163.com

Key words: cathepsin $\mathrm{K}$, cystatin $\mathrm{C}$, atherosclerosis, plaque instability and positive remodeling (2-4), is the final event that precipitates coronary thrombosis and occlusion in about two-thirds of cases of sudden cardiac arrest (5-8). Understanding the pathophysiology of atherosclerosis and the progression of atherosclerotic plaques from stable to unstable is a key focus of ongoing cardiovascular research (1). However, the complex molecular and cellular mechanisms that underlie plaque instability are not completely understood, which is a barrier to the development of interventions aimed at treating and preventing plaque instability (9). Therefore, identifying the mechanisms underlying plaque instability may help to prevent and treat plaque vulnerability.

Plaque instability may be caused by extracellular matrix degradation due to excessive proteolysis (4). Cysteinyl cathepsins regulate cysteine protease activity in all nucleated cells and maintain a balance between the production and degradation of extracellular matrix. Cystatin $\mathrm{C}$ (Cys C) is an important endogenous inhibitor of cathepsins B, H, K, L and S, which may cause the rupture of plaques; cathepsin K (Cat K) is an attractive therapeutic target owing to its collagenase and elastase activity (10). Selective inhibitors of Cat K, such as Odanacatib, have already been used in phase III clinical trials in patients with osteoporosis $(10,11)$ It was demonstrated that Cat $\mathrm{K}$ knockout reduced plaque formation and complexity in mice (12). Cat $\mathrm{K}$ was the first cathepsin identified in human atherosclerosis over a decade ago (13). Subsequently, the expression of Cat B, F, L and $\mathrm{S}$ in plaques was detected, suggesting that cathepsins have a negative effect on plaque stability.

The increased expression of Cat $\mathrm{K}$ in macrophages and vascular smooth muscle cells in the atherosclerotic plaque contrasts with its minimal expression in healthy arteries (13). In addition, Cat $\mathrm{K}$ expression in the endothelium is positively associated with elastic lamina fragmentation, as indicated by immunohistochemical analysis of frozen sections of atherosclerotic human coronary arteries in patients undergoing heart transplant (14). Increased serum levels of Cat K are closely associated with coronary artery disease (CAD) and circulating Cat K may be a useful biomarker for CAD $(15,16)$. However, the expression of $\mathrm{Cat} \mathrm{K}$ in unstable atherosclerotic plaques in humans and its association with plaque instability remains unknown.

The current study assessed Cat $\mathrm{K}$ expression in stable and unstable atherosclerotic plaques sourced from the human 
popliteal artery to determine the association of Cat $\mathrm{K}$ and Cys $\mathrm{C}$ expression with plaque instability. The regulatory mechanism for $\mathrm{Cat} \mathrm{K}$ expression in plaques was investigated to identify potential therapeutic targets.

\section{Materials and methods}

Patients and tissue sampling. Between March 2008 and March 2010, 18 patients [14 men (age range, 48-77 years) and 4 women (age range 58-81 years)] with lower extremity arteriosclerosis obliterans undergoing amputation and 10 patients [6 men (age range, 31-42 years) and 4 women (age range, 33-38 years)] undergoing subtotal gastrectomy at the First Hospital of Jilin University (Jilin, China) were enrolled in the current study. All patients in the control group were determined to not exhibit the disease (osteoporosis or rheumatoid arthritis) and were ages between 18 and 85 years old. Popliteal artery specimens with atherosclerotic plaques were collected from the amputated lower extremities. Mesenteric artery specimens without atherosclerotic plaques were sourced from the subtotal gastrectomy specimens. All arterial specimens were washed in phosphate-buffered saline (PBS) and longitudinally sectioned into two halves. One half was immediately frozen and stored at $-80^{\circ} \mathrm{C}$ prior to RNA extraction; the other half was fixed in $4 \%$ paraformaldehyde for $24 \mathrm{~h}$ at room temperature prior to hematoxylin and eosin (H\&E) staining and immunohistochemical analysis. Sample collections and operations were performed in accordance with the ethical guidelines of the 2013 Declaration of Helsinki and ethical approval for the current study was obtained from the Ethics Committee at Jilin University (Jilin, China). Written informed consent was obtained from all patients prior to their enrollment.

$H \& E$ staining. Morphological characteristics of the atherosclerotic plaque were detected by $\mathrm{H} \& \mathrm{E}$ staining and plaques were subsequently classified as stable or unstable. Fixed samples were demineralized with $12.5 \%$ disodium ethylenediamine tetraacetic acid (EDTA) at room temperature for 1 week and $3-\mu \mathrm{m}$ thick sections were prepared. H\&E-stained sections were observed using a light microscope (BX51; Olympus Corp., Tokyo, Japan) at magnifications of x40, x100 or x400. All examinations were conducted independently by two investigators. Atherosclerotic plaques with a core lipid pool accounting for $>40 \%$ of the total plaque area were classified as unstable; all other plaques were classified as stable (17).

Immunohistochemical analyses. The expression of Cat $\mathrm{K}$ and $\mathrm{Cys} \mathrm{C}$ in plaques was determined following immunohistochemical examinations. Antibodies against human $\mathrm{Cat} \mathrm{K}$ (bs-1611R) were purchased from Beijing Biosynthesis Biotechnology Co., Ltd. (Beijing, China) and those against human Cys C (IT-010-006) were purchased from Abcam (Cambridge, MA, US). Arterial sections that were $3 \mu \mathrm{m}$ thick were fixed in $5 \%$ formaldehyde for 7 days at room temperature and serially dehydrated in successively decreasing concentrations of ethanol $(100,95,90,80$ and $70 \%)$. Following dehydration, sections were attached to poly-1-lysine glass slides, rinsed with PBS (0.01 M, pH 7.4) and treated with hydrogen peroxide to deactivate endogenous peroxides. Sections were blocked for $1 \mathrm{~h}$ at room temperature with $1 \%$ bovine serum albumin obtained from Beijing Dingguo Changsheng Biotechnology, Co., Ltd. (Beijing, China) in PBS, followed by incubation with the antibodies against $\mathrm{Cat} K$ and Cys $\mathrm{C}$ (1:100 dilution) for $12 \mathrm{~h}$ at $44^{\circ} \mathrm{C}$. Following rinsing in PBS 3 times, specimens were incubated with biotinylated secondary antibody at a dilution of 1:100 (SP9710; Fuzhou Maixin Biotech. Co., Ltd, Fuzhou, China) for $10 \mathrm{~min}$ at $37^{\circ} \mathrm{C}$. Specimens were then incubated with horseradish peroxidase conjugated streptavidin solution for $10 \mathrm{~min}$ at $37^{\circ} \mathrm{C}$, followed by incubation with diaminobenzidine for $1-2 \mathrm{~min}$ at $37^{\circ} \mathrm{C}$. Stained sections were counterstained with $\mathrm{H} \& \mathrm{E}$ and examined under a light microscope at magnifications of $x 40, x 100$ and $x 400$. The staining density for Cat K and $\mathrm{Cys} \mathrm{C}$ immunopositive nuclei and cytoplasm were examined in five randomly selected fields using gray scale analysis (Images Advanced 3.2 systems; Motic Co., Xiamen, China).

Semi-quantitative reverse transcription polymerase chain reaction ( $R T-P C R)$. The expression of Cat $\mathrm{K}$ mRNA was measured by semi-quantitative RT-PCR. Total RNA was extracted from plaque tissues using TRIzol (Invitrogen; Thermo Fisher Scientific, Inc., Waltham, MA, USA), following the manufacturer's instructions. Total RNA was determined by the ratio of optical density readings at 260 and $280 \mathrm{~nm}$ and integrity of the specimen was assessed using $1.8 \%$ agarose gel electrophoresis. Rat GAPDH, a housekeeping gene, was used as an internal reference. The sequences of the primers used (Sangon Biotech Co., Ltd., Shanghai, China) and parameters of Cat K and GAPDH for amplification are listed in Table I.

RT-PCR was performed using the Gene Amplify PCR System (Takara Bio, Inc., Otsu, Japan). Amplification specimens were subjected to electrophoresis on a $1.8 \%$ agarose gel (Beijing Dingguo Changsheng Biotechnology, Co., Ltd., Beijing, China), visualized following staining with $0.5 \mathrm{~g} / \mathrm{ml}$ ethidium bromide (Beijing Dingguo Biotechnology, Co., Ltd.) and images were acquired. An image analysis system (Tanon-4200; Tanon Science \& Technology, Co., Ltd., Shanghai, China) was used to scan gel images and the relative intensity of specific PCR bands was determined in relation to GAPDH bands from the same sample.

Statistical analyses. Data were expressed as the mean \pm standard deviation. One-way analysis of variance followed by Bonferroni's multiple comparison tests was used to assess between-group differences. Spearman's correlation was used to assess the correlation between the expression of Cat $\mathrm{K}$ and Cys C. All statistical analyses were assessed using SPSS 19.0 (IBM Corp., Armonk, NY, USA) and $\mathrm{P}<0.05$ was considered to indicate a statistically significant difference.

\section{Results}

Plaque morphology. Six specimens of atherosclerotic tissue were classified as stable plaque and 14 were classified as unstable plaque. Orderly arrangements of endothelial cells and smooth endomembranes were observed in mesenteric artery specimens from controls. No thickening of the media or presence of a lipid core was observed (Fig. 1A).

In stable atherosclerotic plaques from popliteal arteries, the proliferation of endothelial cells and internal elastic lamina was detected (Fig. 1B). In unstable popliteal artery plaques, 
Table I. Primers and parameters of Cathepsin K and GAPDH for amplification.

Cathepsin K

\begin{tabular}{lll}
\hline Forward (5'-3') & CAGTGAAGAGGTGGTTCAGA & GGGTGATGCTGGTGCTGAGTATGT \\
Reverse (5'-3') & TTCCATCTCGGGGTCTGAGA & AAGAATGGGTGTTGCTGTTGAAGTC \\
Fragment length (bps) & 109 & 617 \\
Cycles & $94^{\circ} \mathrm{C}, 30 \mathrm{sec} ; 52^{\circ} \mathrm{C}, 30 \mathrm{sec} ; 72^{\circ} \mathrm{C}, 30 \mathrm{sec} ; 30 \mathrm{cycles}$ & $94^{\circ} \mathrm{C}, 30 \mathrm{sec} ; 57^{\circ} \mathrm{C}, 30 \mathrm{sec} ; 72^{\circ} \mathrm{C}, 30 \mathrm{sec} ; 28 \mathrm{cycles}$ \\
Final extension & $72^{\circ} \mathrm{C} 10 \mathrm{~min}$ & $72^{\circ} \mathrm{C} 10 \mathrm{~min}$ \\
\hline
\end{tabular}

A

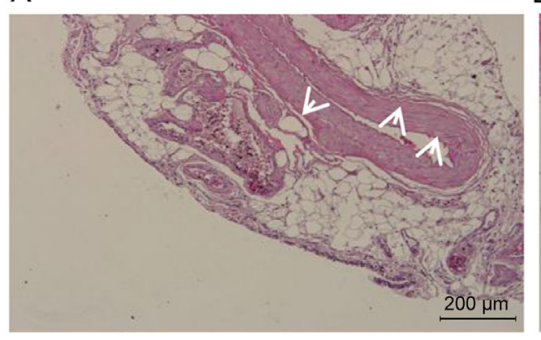

B

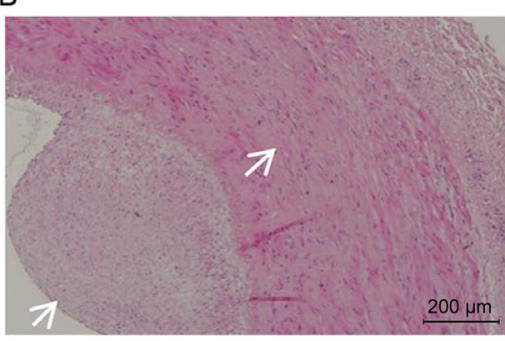

C

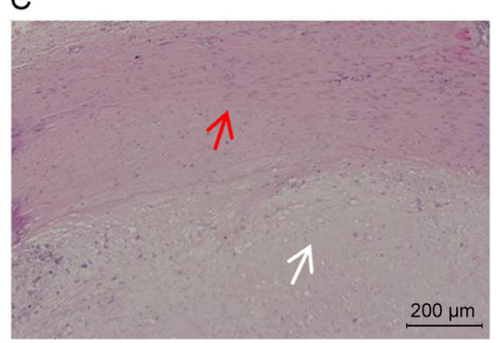

Figure 1. Histopathological characteristics of arteries with and without atherosclerotic plaques. (A) Representative images of control mesenteric artery ( $=10$; magnification, x100) presenting the structural integrity of the vascular wall, smooth intima, arranged endothelial cells, smooth muscle cells and lack of medial hyperplasia, and outer layer of loose connective tissue. (B) Representative images of popliteal artery with stable plaques (n=6; magnification, x100) exhibiting eccentric intimal thickening and no clear lipid core. The plaque is composed of foam cells with pink lipid deposition and the internal elastic lamina is clear. The media exhibited SMC proliferation, disordered arrangements and inflammatory cell infiltration. (C) Representative images of the popliteal artery with unstable plaque $(\mathrm{n}=14$; magnification, $\mathrm{x} 100)$. Numerous uniform pink lipid deposits (indicated by white arrow), formation of cavity following dissolution of lipids, calcium deposits (purple), foam cells and destruction of the internal elastic lamina are visible in the intima. The red arrow indicates SMC proliferation, disordered arrangement, foam cell infiltration, calcium deposits, lipid deposition and the presence of cholesterol crystals in the media. SMC, smooth muscle cell.

intimal thickening and the presence of large lipid cores $(>40 \%$ of the total plaque area) were observed. The proliferation of endothelial cells, presence of foam cells and intimal lipid deposits were also detected. Furthermore, the infiltration of inflammatory cells, presence of cholesterol crystals, destruction of internal elastic lamina along with large lipid deposits and hyperplasia of vascular smooth muscle cells were observed (Fig. 1C).

Cat $K$ expression in atherosclerotic plaques. Positive brown staining for Cat $\mathrm{K}$ was observed in atherosclerotic plaques. In the mesenteric artery specimens from controls, there was weak positive staining for $\mathrm{Cat} \mathrm{K}$ in the intimal and medial cells (Fig. 2A). In the stable popliteal artery plaques, positive staining for Cat $\mathrm{K}$ (indicated by the presence of brown particles) was observed in the plaque, intima and media (Fig. 2B). In the unstable popliteal artery plaques, there was strong positive staining for $\mathrm{Cat} \mathrm{K}$ in the plaque, intima and media, indicated by the presence of brown flakiness (Fig. 2C).

Gray scale analyses indicated that the intensity of positive staining for Cat $\mathrm{K}$ in stable $(161.75 \pm 1.38)$ and unstable (163.9 \pm 1.331$)$ plaques was significantly greater than that in normal control mesenteric arteries $(120.7 \pm 4.18$; both $\mathrm{P}<0.05)$. Furthermore, the intensity of positive staining for Cat $\mathrm{K}$ in unstable plaque specimens was significantly greater than that of stable plaque specimens ( $\mathrm{P}<0.05$; Fig. 2D).

Cat $K$ mRNA expression in unstable atherosclerotic plaques. Levels of Cat K mRNA were significantly upregulated in stable and unstable plaques compared with normal mesenteric artery specimens (both $\mathrm{P}<0.05$ ). Furthermore, levels of Cat $\mathrm{K}$ mRNA in unstable plaques were significantly greater than in stable plaques $(\mathrm{P}<0.05$; Fig. 3$)$.

Cys $C$ expression in unstable atherosclerotic plaques. The positive expression of $\mathrm{Cys} \mathrm{C}$ was indicated by deep brown staining. In the mesenteric arteries from controls, there was positive staining for $\mathrm{Cat} \mathrm{K}$ in the intimal and medial cells (Fig. 4A). In stable and unstable atherosclerotic plaques from popliteal arteries, positive staining for Cys $\mathrm{C}$ was observed in the plaque, intima and media (brown particles; Fig. 4B and C).

Grayscale analyses demonstrated that the intensity of positive staining for Cys C in stable (127.20 2.269$)$ and unstable $(121.9 \pm 1.732)$ plaque specimens was significantly higher than that in normal mesenteric artery specimens (137.8 \pm 3.323 ; $\mathrm{P}<0.05$ for both). In addition, the intensity of staining in unstable plaque specimens was significantly higher than that of the stable plaque specimens $(\mathrm{P}<0.05$; Fig. 4D).

The association between the expression of Cat $\mathrm{K}$ and Cys $\mathrm{C}$ in unstable popliteal artery plaques was assessed using Spearman's correlation analysis. In unstable plaques, an inverse association was observed between the expression of Cat $\mathrm{K}$ and Cys C ( $\mathrm{r}=-0.9228, \mathrm{P}=0.0011)$.

\section{Discussion}

The current study investigated the association between Cat $\mathrm{K}$ and plaque instability in human atherosclerotic plaques. The 


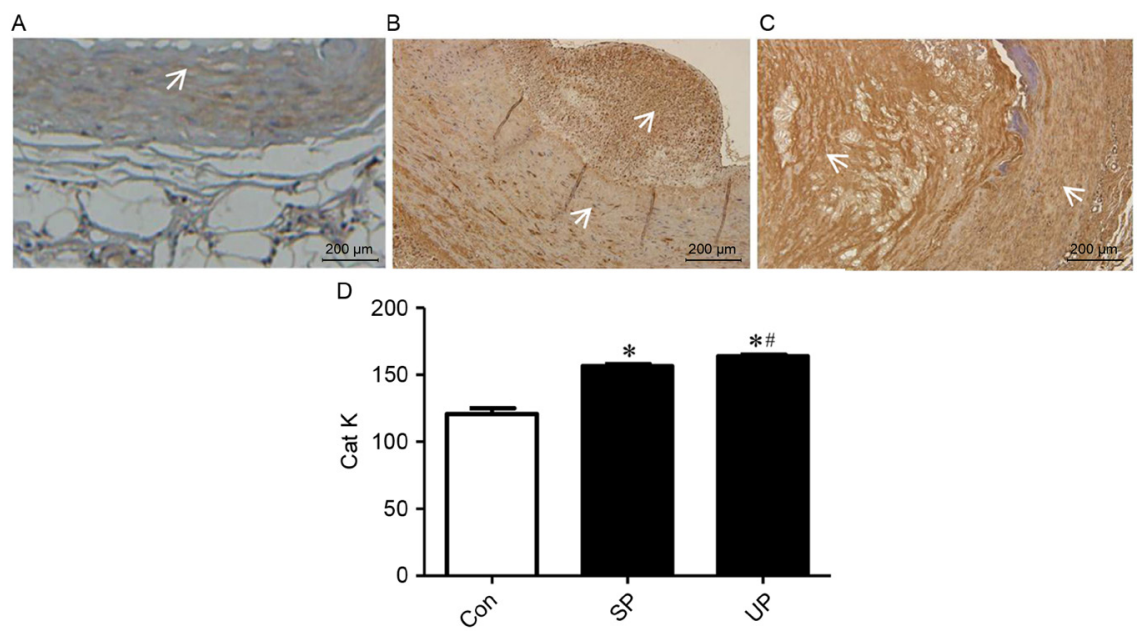

Figure 2. Cat $\mathrm{K}$ expression in arterial specimens with and without atherosclerotic plaques. (A) Representative image of mesenteric artery specimens from controls ( $\mathrm{n}=10$; magnification, $\mathrm{x} 100)$ indicating mild positive Cat $\mathrm{K}$ staining in the intima and uniform brown Cat $\mathrm{K}$ staining in the cytoplasm of SMCs in media. (B) Representative image of stable popliteal artery plaque ( $\mathrm{n}=6$; magnification, $\mathrm{x} 100)$ indicating foam cell infiltration in the intima and plaque, strongly positive Cat $\mathrm{K}$ staining (brown granular) in the intima and plaque and slight granular positive Cat $\mathrm{K}$ staining in the media. (C) Representative image of unstable popliteal artery plaque ( $\mathrm{n}=14$, magnification, $\mathrm{x} 100)$ presenting high intensity of Cat $\mathrm{K}$ staining in the plaque, intima and media (visible as brown flakiness). Unstable plaques exhibited marked intimal thickening, accumulation of foam cells and brown granular Cat K staining in the foam cells and SMC of media. (D) Statistical analyses of Cat K expression. Positive Cat K gray intensity in unstable plaque specimens was significantly lower than that in the stable plaque specimens. The results presented the expression of Cat $\mathrm{K}$ in the UP compared with the SP. ${ }^{*} \mathrm{P}<0.05$ vs. Con, ${ }^{\text {"P }}<0.05$ vs. SP. Cat K, cathepsin K; Con, control group; SP, stable plaque group; UP, unstable plaque group; SMC, smooth muscle cell.

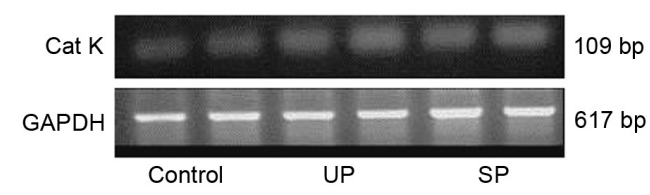

B

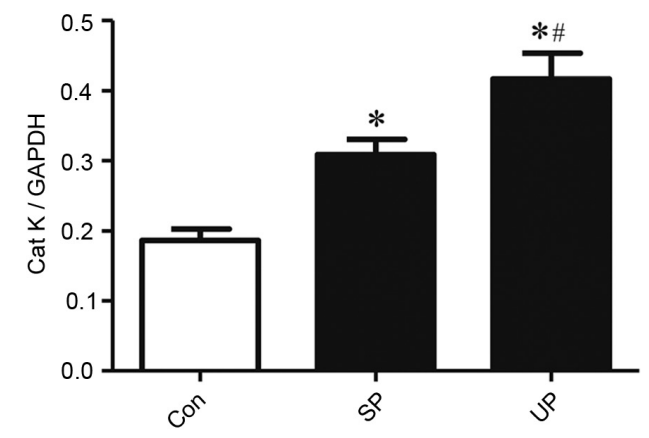

Figure 3. Levels of Cat K mRNA in arterial specimens with and without atherosclerotic plaques. (A) Representative image of Cat K and GAPDH following agarose gel electrophoresis. Lane 1, DNA markers; lanes 2 and 3, Con; lanes 4 and 5, UP; lanes 6 and 7, SP. (B) Statistical analyses of Cat K mRNA expression. mRNA expression in unstable plaques was significantly greater than in stable plaques. ${ }^{*} \mathrm{P}<0.05$ vs. Con; ${ }^{\#} \mathrm{P}<0.05$ vs. SP. Cat $\mathrm{K}$, cathepsin $\mathrm{K}$; Con, control group; SP, stable plaque group; UP, unstable plaque group; bp, base pairs.

expression of Cat $\mathrm{K}$ mRNA and protein in stable and unstable popliteal artery plaques was greater than that of control mesenteric artery specimens. Furthermore, the expression of Cat $\mathrm{K}$ mRNA and protein in unstable plaque was significantly higher than in stable plaques. By contrast, a decrease in the expression of Cys $C$ was detected in stable and unstable plaques and the expression of Cys $\mathrm{C}$ in unstable plaques was significantly lower than in stable plaques.
Notably, the decrease in Cys $\mathrm{C}$ expression in the atherosclerotic plaques was negatively correlated with an increase in Cat $\mathrm{K}$ expression. These results suggest that the elevation of Cat $\mathrm{K}$ expression may stimulate the progression of atherosclerotic plaques from stable to unstable. Low expression of Cys $\mathrm{C}$ is associated with the high expression of Cat $\mathrm{K}$, therefore Cat $\mathrm{K}$ and Cys $\mathrm{C}$ may be potential therapeutic targets for preventing and treating unstable atherosclerotic plaques.

Non-ruptured plaques with a large lipid-rich necrotic core and thinned out collagen-depleted fibrous cap, which exhibit smooth muscle cell proliferation, intimal and adventitial inflammation and microcalcification are considered to be unstable or vulnerable plaques, owing to their heightened risk of rupture (4). The existence of some of these pathological features has been determined in previous clinical studies $(18,19)$. Atherosclerotic plaque specimens with a core lipid pool accounting for $>40 \%$ of the total plaque area is classified as unstable (20). Furthermore, unstable atherosclerotic plaques exhibit several hallmark features. Such plaques are composed of abundant intimal lipid deposits, proliferating endothelial cells, foam cells, inflammatory infiltrate and cholesterol crystals, and are marked by destruction of internal elastic lamina, large lipid deposits and hyperplasia of vascular smooth muscle cells in the media (20). All these signs are indicative of unstable atherosclerotic plaques.

In the current study, it was demonstrated that the expression of Cat K mRNA and protein in unstable plaques was significantly higher than in stable plaques. Cat $\mathrm{K}$ is a papain-like cysteine peptidase. Its physiological role is reflected in its distinctive cleavage model for type I collagen molecules, which is different from that of the other endogenous collagenases (11). The specific biological activity of Cat $\mathrm{K}$ is to cleave the triple helix of collagen molecules at multiple positions (11). In addition, Cat $\mathrm{K}$ is a powerful elastinolytic enzyme $(10,11)$. Cat $\mathrm{K}$, secreted by macrophages along with several other cysteine 


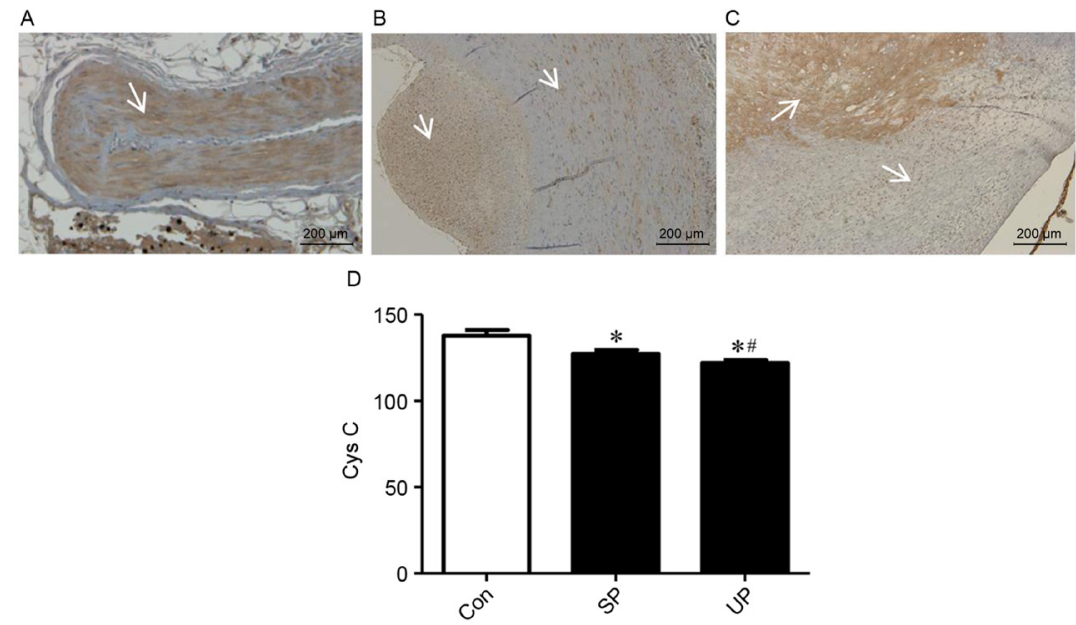

Figure 4. Expression of Cys $\mathrm{C}$ protein in arterial specimens with and without atherosclerotic plaques. (A) Representative image of mesenteric artery specimens from controls ( $\mathrm{n}=10$; magnification, $\mathrm{x} 100$ ) indicating abundant positive staining for Cat $\mathrm{K}$ in the intimal and medial cells (deep brown flakiness). (B) Representative image of stable popliteal artery plaque $(\mathrm{n}=6$; magnification, $\mathrm{x} 100)$ indicating foam cell infiltration, dark brown granular Cys $\mathrm{C}$ staining of the intimal layer and mild Cys C staining (deep brown particles) in the media. (C) Representative image of unstable popliteal artery plaque (n=14, magnification, x100). Deep brown granular Cys C staining of foam cells in the lipid core and intimal layer as well as destruction of media was detected. (D) Statistical analyses of Cys $\mathrm{C}$ protein expression. Positive staining for Cys $\mathrm{C}$ in unstable plaque specimens was significantly higher than that in the stable plaque specimens. The results indicate the downregulation of Cys C expression in unstable plaques compared with stable plaques. ${ }^{*} \mathrm{P}<0.05$ vs. Con; ${ }^{*} \mathrm{P}<0.05$ vs. stable plaque group. Cys C, cystatin C; Cat K, cathepsin K; Con, control group; SP, stable plaque group; UP, unstable plaque group.

cathepsins, degrades the vascular elastic lamina (21). The involvement of Cat $\mathrm{K}$ in macrophage-mediated extracellular elastin degradation (22) and in the degradation of extracellular matrix induced by human macrophage foam cells (23) has been demonstrated. Notably, upregulation of Cat $\mathrm{K}$ is also associated with atherosclerosis (13). In apolipoprotein E-deficient $\left(\mathrm{apoE}^{-/}\right)$mice, a murine model with spontaneous atherosclerosis, Cat $\mathrm{K}$ deficiency prevented atherosclerotic plaque progression and the induction of plaque fibrosis $(24,25)$. Furthermore, in apo: $\mathrm{E}^{-/}$mice, Cat $\mathrm{K}$ deficiency is associated with a reduction in the size of atherosclerotic plaques and improved structural integrity of the tunica media, as well as elastic lamina of brachiocephalic arteries (26). Increased Cat $\mathrm{K}$ expression in atherosclerotic plaques (13) and elevated serum levels of Cat $\mathrm{K}$ are closely associated with coronary artery disease $(15,16)$. These results suggest that the upregulation of $\mathrm{Cat} \mathrm{K}$ in unstable atherosclerotic plaque may accelerate atherogenesis and induce plaque instability.

The accumulation of oxidized lipids and apoptotic cells in atherosclerotic lesions contributes to plaque rupture and its associated clinical complications (24). Cys C, a small protein secreted by almost all organs and found in biological fluids, including plasma, is the most plentiful and potent endogenous inhibitor of cathepsins B, H, K, L and S (27) and inhibits the activity of lysosomal cathepsins, such as Cat K. Cys $\mathrm{C}$ also serves a regulatory role in autophagy, which protects against the apoptosis induced by 7 -oxysterols by preventing lipid accumulation (28). In addition, it modulates inflammatory responses. These functions are closely associated with apoptosis and plaque destabilization in human atherosclerosis.

In the current study, the elevated expression of Cat $\mathrm{K}$ and downregulation of $\mathrm{Cys} \mathrm{C}$ in unstable plaques was detected. Atherosclerotic mice (apoE ${ }^{-1}$ mice) deficient in Cys C exhibited increased degradation of elastic lamina as well as the formation of larger plaques $(29,30)$. Additionally, it was demonstrated that in human atherosclerotic arteries, the expression of Cys C is severely reduced in plaques exhibiting high expression of Cat K (31). In growing and ruptured abdominal aortic aneurysms, levels of Cat $\mathrm{K}$ mRNA, protein and protease activity were elevated, whereas levels of Cys $C$ protein were markedly reduced (32). Similar alterations in Cat $\mathrm{K}$ and Cys $\mathrm{C}$ expression were reported in the later stages of cerebral aneurysm progression (33). Furthermore, the expression of Cys $\mathrm{C}$ and Cat $\mathrm{K}$ were significantly decreased and increased respectively, during acute myocardial infarction (34). Additionally, the expression of Cys $\mathrm{C}$ was elevated in the brains of Cat $\mathrm{K}$ deficient mice (35). These results indicate that Cat $\mathrm{K}$ expression is inversely associated with Cys $\mathrm{C}$ expression, which may be inherently associated with atherosclerotic instability.

There were a number of limitations of the current study. Only the expression of Cat $\mathrm{K}$ and Cys $\mathrm{C}$ were assessed; their activities were not included in the scope of the study. Additionally, the dynamic interplay between Cat $\mathrm{K}$ and Cys $\mathrm{C}$ in the transformation of atherosclerotic plaques from stable to unstable and the underlying mechanism of action require further clarification. Furthermore, it remains unknown whether inhibiting $\mathrm{Cat} \mathrm{K}$ and/or activating Cys $\mathrm{C}$ are able to attenuate or reverse plaque transformation.

In conclusion, the current study demonstrated that Cat $\mathrm{K}$ expression in unstable human popliteal artery plaques was negatively correlated with Cys $\mathrm{C}$ expression. The interplay between Cat $\mathrm{K}$ and Cys $\mathrm{C}$ expression may serve a fundamental role in the progression of plaques from stable to unstable. Therefore, Cat $\mathrm{K}$ and Cys $\mathrm{C}$ may be potential targets to prevent and treat unstable atherosclerotic plaques.

\section{Acknowledgements}

The present study was supported by the National Natural Science Foundation of China (no. 51372096) and the Province Natural Science Foundation of Jilin, China (no. 201015143). 


\section{References}

1. Silvestre-Roig C, de Winther MP, Weber C, Daemen MJ, Lutgens E and Soehnlein O: Atherosclerotic plaque destabilization: Mechanisms, models, and therapeutic strategies. Circ Res 114: 214-226, 2014

2. Rathore S, Terashima M, Matsuo H, Kinoshita Y, Kimura M, Tsuchikane E, Nasu K, Ehara M, Asakura Y, Katoh O and Suzuki T: Association of coronary plaque composition and arterial remodelling: A optical coherence tomography study. Atherosclerosis 221: 405-415, 2012.

3. Narula J, Nakano M, Virmani R, Kolodgie FD, Petersen R, Newcomb R, Malik S, Fuster V and Finn AV: Histopathologic characteristics of atherosclerotic coronary disease and implications of the findings for the invasive and noninvasive detection of vulnerable plaques. J Am Coll Cardiol 61: 1041-1051, 2013.

4. Shah PK: Biomarkers of plaque instability. Curr Cardiol Rep 16: $547,2014$.

5. Davies MJ and Thomas A: Thrombosis and acute coronary-artery lesions in sudden cardiac ischemic death. N Engl J Med 310: $1137-1140,1984$

6. Gorlin R, Fuster V and Ambrose JA: Anatomic-physiologic links between acute coronary syndromes. Circulation 74: 6-9, 1986.

7. Thompson PL, Nidorf SM and Eikelboom J: Targeting the unstable plaque in acute coronary syndromes. Clin Ther 35: 1099-1107, 2013.

8. Niccoli G, Liuzzo G, Montone RA and Crea F: Advances in mechanisms, imaging and management of the unstable plaque. Atherosclerosis 233: 467-477, 2014.

9. Andreou I, Antoniadis AP, Shishido K, Papafaklis MI, Koskinas KC, Chatzizisis YS, Coskun AU, Edelman ER, Feldman CL and Stone PH: How do we prevent the vulnerable atherosclerotic plaque from rupturing? Insights from in vivo assessments of plaque, vascular remodeling, and local endothelial shear stress. J Cardiovasc Pharmacol Ther 20: 261-275, 2015

10. Newby AC: Proteinases and plaque rupture: Unblocking the road to translation. Curr Opin Lipidol 25: 358-366, 2014.

11. Novinec $\mathrm{M}$ and Lenarčič B: Cathepsin K: A unique collagenolytic cysteine peptidase. Biol Chem 394: 1163-1179, 2013.

12. Cheng XW, Huang Z, Kuzuya M, Okumura K and Murohara T: Cysteine protease cathepsins in atherosclerosis-based vascular disease and its complications. Hypertension 58: 978-986, 2011.

13. Sukhova GK, Shi GP, Simon DI, Chapman HA and Libby P Expression of the elastolytic cathepsins $\mathrm{S}$ and $\mathrm{K}$ in human atheroma and regulation of their production in smooth muscle cells. J Clin Invest 102: 576-583, 1998.

14. Platt MO, Ankeny RF, Shi GP, Weiss D, Vega JD, Taylor WR and Jo H: Expression of cathepsin $\mathrm{K}$ is regulated by shear stress in cultured endothelial cells and is increased in endothelium in human atherosclerosis. Am J Physiol Heart Circ Physiol 292: H1479-H1486, 2007.

15. Cheng XW, Kikuchi R, Ishii H, Yoshikawa D, Hu L, Takahashi R, Shibata R, Ikeda N, Kuzuya M, Okumura K and Murohara T: Circulating cathepsin $\mathrm{K}$ as a potential novel biomarker of coronary artery disease. Atherosclerosis 228: 211-216, 2013.

16. Li X, Li Y, Jin J, Jin D, Cui L, Li X, Rei Y, Jiang H, Zhao G, Yang $\mathrm{G}$, et al: Increased serum cathepsin $\mathrm{K}$ in patients with coronary artery disease. Yonsei Med J 55: 912-919, 2014.

17. de Boer OJ, van der Wal AC, Teeling P and Becker AE: Leucocyte recruitment in rupture prone regions of lipid-rich plaques: A prominent role for neovascularization? Cardiovasc Res 41: 443-449, 1999.

18. Bentzon JF, Otsuka F, Virmani R and Falk E: Mechanisms of plaque formation and rupture. Circ Res 114: 1852-1866, 2014.

19. Garcia-Garcia HM, Jang IK, Serruys PW, Kovacic JC, Narula J and Fayad ZA: Imaging plaques to predict and better manage patients with acute coronary events. Circ Res 114: 1904-1917, 2014.
20. Xu B, Zhao H, Wang S, Sun X and Qin X: Increased ADRP expression in human atherosclerotic lesions correlates with plaque instability. Int J Clin Exp Med 8: 5414-5421, 2015.

21. Punturieri A, Filippov S, Allen E, Caras I, Murray R, Reddy V and Weiss SJ: Regulation of elastinolytic cysteine proteinase activity in normal and cathepsin K-deficient human macrophages. J Exp Med 192: 789-799, 2000.

22. Yasuda Y, Li Z, Greenbaum D, Bogyo M, Weber E and Brömme D: Cathepsin V, a novel and potent elastolytic activity expressed in activated macrophages. J Biol Chem 279: 36761-36770, 2004.

23. Barascuk N, Skjøt-Arkil H, Register TC, Larsen L, Byrjalsen I, Christiansen C and Karsdal MA: Human macrophage foam cells degrade atherosclerotic plaques through cathepsin $\mathrm{K}$ mediated processes. BMC Cardiovasc Disord 10: 19, 2010.

24. Lutgens E, Lutgens SP, Faber BC, Heeneman S, Gijbels MM, de Winther MP, Frederik P, van der Made I, Daugherty A, Sijbers AM, et al: Disruption of the cathepsin K gene reduces atherosclerosis progression and induces plaque fibrosis but accelerates macrophage foam cell formation. Circulation 113: 98-107, 2006.

25. Lutgens SP, Kisters N, Lutgens E, van Haaften RI, Evelo CT, de Winther MP, Saftig P, Daemen MJ, Heeneman S and Cleutjens KB: Gene profiling of cathepsin K deficiency in atherogenesis: Profibrotic but lipogenic. J Pathol 210: 334-343, 2006.

26. Samokhin AO, Wong A, Saftig P and Brömme D: Role of cathepsin $\mathrm{K}$ in structural changes in brachiocephalic artery during progression of atherosclerosis in apoE-deficient mice. Atherosclerosis 200: 58-68, 2008.

27. Lafarge JC, Naour N, Clement K and Guerre-Millo M: Cathepsins and cystatin $\mathrm{C}$ in atherosclerosis and obesity. Biochimie 92: 1580-1586, 2010.

28. Li W, Sultana N, Siraj N, Ward LJ, Pawlik M, Levy E, Jovinge S, Bengtsson E and Yuan XM: Autophagy dysfunction and regulatory cystatin $\mathrm{C}$ in macrophage death of atherosclerosis. J Cell Mol Med 20: 1664-1672, 2016.

29. Sukhova GK, Wang B, Libby P, Pan JH, Zhang Y, Grubb A, Fang K, Chapman HA and Shi GP: Cystatin C deficiency increases elastic lamina degradation and aortic dilatation in apolipoprotein E-null mice. Circ Res 96: 368-375, 2005.

30. Bengtsson E, To F, Håkansson K, Grubb A, Brånén L, Nilsson J and Jovinge S: Lack of the cysteine protease inhibitor cystatin $\mathrm{C}$ promotes atherosclerosis in apolipoprotein E-deficient mice. Arterioscler Thromb Vasc Biol 25: 2151-2156, 2005.

31. Shi GP, Sukhova GK, Grubb A, Ducharme A, Rhode LH, Lee RT, Ridker PM, Libby P and Chapman HA: Cystatin C deficiency in human atherosclerosis and aortic aneurysms. J Clin Invest 104: 1191-1197, 1999.

32. Abdul-Hussien H, Soekhoe RG, Weber E, von der Thüsen JH, Kleemann R, Mulder A, van Bockel JH, Hanemaaijer R and Lindeman JH: Collagen degradation in the abdominal aneurysm: A conspiracy of matrix metalloproteinase and cysteine collagenases. Am J Pathol 170: 809-817, 2007.

33. Aoki T, Kataoka H, Ishibashi R, Nozaki $\mathrm{K}$ and Hashimoto $\mathrm{N}$ : Cathepsin B, K and $\mathrm{S}$ are expressed in cerebral aneurysms and promote the progression of cerebral aneurysms. Stroke 39: 2603-2610, 2008

34. Shalia KK, Mashru MR, Shah VK, Soneji SL and Payannavar S: Levels of cathepsins in acute myocardial infarction. Indian Heart J 64: 290-294,2012.

35. Dauth S, Sirbulescu RF, Jordans S, Rehders M, Avena L, Oswald J, Lerchl A, Saftig P and Brix K: Cathepsin K deficiency in mice induces structural and metabolic changes in the central nervous system that are associated with learning and memory deficits. BMC Neurosci 12: 74, 2011 